Use of granulocyte colony-stimulating factor and risk of relapse in pediatric patients treated for acute myeloid leukemia according to NOPHO-AML 2004 and DB AML-01

\author{
Lohmann, Ditte J. A.
}

2019-06

Lohmann , D J A , Asdahl , P H , Abrahamsson , J , Ha , S-Y, Jonsson , O G , Kaspers , G J L , Koskenvuo , M , Lausen , B , De Moerloose , B , Palle , J , Zeller , B \& Hasle , H 2019 , ' Use of granulocyte colony-stimulating factor and risk of relapse in pediatric patients treated for acute myeloid leukemia according to NOPHO-AML 2004 and DB AML-01 ', Pediatric Blood \& Cancer , vol. 66 , no. 6 , 27701 . https://doi.org/10.1002/pbc.27701

http://hdl.handle.net/10138/312882

https://doi.org/10.1002/pbc.27701

draft

Downloaded from Helda, University of Helsinki institutional repository.

This is an electronic reprint of the original article.

This reprint may differ from the original in pagination and typographic detail.

Please cite the original version. 


\title{
Use of granulocyte colony-stimulating factor and risk of relapse in pediatric patients treated for acute myeloid leukemia according to NOPHO-AML 2004 and DB AML-01
}

\author{
Ditte J.A. Løhmann ${ }^{1}$ (iD $\quad$ | Peter H. Asdahl ${ }^{2}$ | Jonas Abrahamsson ${ }^{3}$ | Shau-Yin $\mathrm{Ha}^{4}$ | \\ Ólafur G. Jónsson $^{5}$ ｜ Gertjan J. L. Kaspers ${ }^{6,7,8}$ | Minna Koskenvuo ${ }^{9}$ | Birgitte Lausen ${ }^{10}$ \\ Barbara De Moerloose $^{11}$ | Josefine Palle ${ }^{12}$ | Bernward Zeller ${ }^{13}$ | Henrik Hasle ${ }^{1}$
}

\begin{abstract}
${ }^{1}$ Department of Pediatrics and Adolescent Medicine, Aarhus University Hospital, Aarhus, Denmark
${ }^{2}$ Department of Hematology, Aarhus University Hospital, Aarhus, Denmark

${ }^{3}$ Institution for Clinical Sciences, Department of Pediatrics, Queen Silvia Children's Hospital, Gothenburg, Sweden

${ }^{4}$ Department of Pediatrics, Queen Mary Hospital and Hong Kong Pediatric Hematology and Oncology Study Group (HKPHOSG), Hong Kong, China

${ }^{5}$ Department of Pediatrics, Landspitali University Hospital, Reykjavik, Iceland

${ }^{6}$ Department of Pediatrics, VU University Medical Center, Amsterdam, The Netherlands

${ }^{7}$ Princess Máxima Center for Pediatric Oncology, Utrecht, The Netherlands

${ }^{8}$ Dutch Childhood Oncology Group, The Hague, The Netherlands

${ }^{9}$ Division of Hematology-Oncology and Stem Cell Transplantation, New Children's Hospital, University of Helsinki and Helsinki University Hospital, Helsinki, Finland

${ }^{10}$ Department of Pediatrics and Adolescent Medicine, Rigshospitalet, University of Copenhagen, Copenhagen, Denmark

${ }^{11}$ Department of Pediatric Hematology-Oncology and Stem Cell Transplantation, Ghent University Hospital, Ghent, Belgium

${ }^{12}$ Department of Woman's and Children's Health, Uppsala University, Uppsala, Sweden

${ }^{13}$ Division of Pediatric and Adolescent Medicine, Oslo University Hospital, Oslo, Norway
\end{abstract}

Correspondence

Ditte J. A. Løhmann, Department of Pediatrics and Adolescent Medicine, Aarhus University Hospital, Palle Juul-Jensens Boulevard 99,

Aarhus N DK-8200, Denmark.

Email: dadolfsen@gmail.com

\section{Funding information}

Børnecancerfonden, Grant/Award Numbers: 2013-12, 2014-10; Kræftens Bekæmpelse, Grant/Award Number: R72-A4681-13-52; Novo Nordisk Foundation, Grant/Award Number: 7941

\begin{abstract}
Background: Supportive-care use of granulocyte colony-stimulating factor (G-CSF) in pediatric acute myeloid leukemia (AML) remains controversial due to a theoretical increased risk of relapse and limited impact on neutropenic complications. We describe the use of G-CSF in patients treated according to NOPHO-AML 2004 and DB AML-01 and investigated associations with relapse.

Procedure: Patients diagnosed with de novo AML completing the first week of therapy and not treated with hematopoietic stem cell transplantation in the first complete remission were included ( $n=367$ ). Information on G-CSF treatment after each course (yes/no) was registered prospectively in the study database and detailed information was gathered retrospectively from each center. Descriptive statistics were used to describe G-CSF use and Cox regression to assess the association between G-CSF and risk of relapse.

Results: G-CSF as supportive care was given to 128 (35\%) patients after 268 (39\%) courses, with a large variation between centers (0-93\%). The use decreased with time-the country-adjusted odds ratio was 0.8 /diagnostic year (95\% confidence interval [Cl] 0.7-0.9). The median daily dose was $5 \mu \mathrm{g} / \mathrm{kg}$ (range 3-12 $\mu \mathrm{g} / \mathrm{kg}$ ) and the median cumulative dose was $75 \mu \mathrm{g} / \mathrm{kg}$ (range 7-1460 $\mu \mathrm{g} / \mathrm{kg}$ ). Filgrastim was used in $82 \%$ of G-CSF administrations and infection was the indication in $44 \%$ of G-CSF administrations. G-CSF was associated with increased risk of relapse-the adjusted hazard ratio was $1.5(95 \% \mathrm{Cl} 1.1-2.2)$.
\end{abstract}


Conclusions: G-CSF as supportive care was used in a third of patients, and use decreased with time. Our results indicate that the use of G-CSF may be associated with an increased risk of relapse.

KEYWORDS

AML, G-CSF, pediatric, relapse

\section{1 | INTRODUCTION}

Treatment for pediatric acute myeloid leukemia ( $\mathrm{AML}$ ) is highly toxic and $5-10 \%$ of patients die as a consequence of treatment. ${ }^{1-7}$ Infections during neutropenia are the most frequent cause of treatmentrelated mortality and a major cause of morbidity. ${ }^{1,2,4,6-8}$ Granulocyte colony-stimulating factor (G-CSF) has been used to decrease time with neutropenia, aiming to reduce severe infections and treatmentrelated mortality. Yet, while G-CSF has been shown to reduce the duration of neutropenia in children treated for cancer, ${ }^{9}$ the use of G-CSF in AML remains controversial. This is due to reports showing that myeloid leukemia cells express G-CSF receptors and that G-CSF can stimulate proliferation of leukemic blasts in vitro. ${ }^{10,11}$ Furthermore, an increased risk of relapse has been shown in patients receiving $\mathrm{G}$ CSF and overexpressing G-CSFR isoform IV. ${ }^{12}$ G-CSF does not seem to reduce microbiologically documented infections or treatment-related mortality. ${ }^{13,14}$ The general use of G-CSF for prevention of infectious complications in children or adults during treatment is currently not recommended. ${ }^{15,16}$

In the last concluded Nordic and Dutch-Belgian pediatric AML protocols (NOPHO-AML 2004 and DB AML-01), G-CSF was not part of standard recommendations but could be administered according to physician preference. In this study, we aimed to describe supportivecare G-CSF use including indications and cumulative doses in children treated according to NOPHO-AML 2004 and DB AML-01. Secondly, we aimed to investigate associations between G-CSF treatment and risk of relapse.

\section{I MATERIALS AND METHODS}

\section{1 | Patients}

Patients treated according to NOPHO-AML 2004 and DB AML-01 were eligible for this study. The protocols were population based in children less than 15 years of age (for some centers, less than 18 years) diagnosed with AML in the five Nordic countries (Denmark, Finland, Iceland, Norway, and Sweden) from 2004 to 2013 (NOPHOAML 2004), in Hong Kong from 2007 to 2013 (NOPHO-AML 2004), in Belgium from 2010 to 2014 (DB AML-01), and the Netherlands from 2010 to 2013 (DB AML-01). Children with Down syndrome, acute promyelocytic leukemia, isolated granulocytic sarcoma, or secondary AML were excluded. Further exclusion criteria for this study were (a) death within the first week after starting treatment and (b) treatment with hematopoietic stem cell transplantation (HSCT) in the first complete remission (CR1).

\section{2 | Protocols}

Protocol details for NOPHO-AML 2004 and DB AML-01 including drug doses have been published previously. ${ }^{17-19}$ The NOPHO-AML 2004 protocol consisted of two induction courses (AIET and AM) and four consolidation courses $\left(\mathrm{HA}_{1} \mathrm{M}, \mathrm{HA}_{2} \mathrm{E} 1, \mathrm{HA}_{3}\right.$, and $\left.\mathrm{HA}_{2} \mathrm{E} 2\right)$ followed by a randomization to gemtuzumab ozogamicin or no further therapy. Children not in remission after two induction courses were given FLAG as a third induction course, whereas those in remission proceeded to consolidation therapy. G-CSF given as a part of FLAG was not considered supportive care, and is therefore not included in this study. Highrisk patients (bone marrow blasts $>15 \%$ after AIET or no remission after second induction course or the presence of KMT2A rearrangements other than $t[9 ; 11][p 21 ; q 23])$ were offered allogeneic HSCT. The following three amendments were made during the protocol period: (1) In 2009, the high-risk criteria were restricted to poor response only, (2) in 2011, FLT3 with internal tandem duplication (FLT3/ITD) without NPM1 mutations became a high-risk criterion, and (3) in 2011, the second induction course was changed from AM to FLA-Dx in patients with $t(8 ; 21)$ or bone marrow blasts $5-15 \%$ on day $15 .^{20}$

The DB AML-01 protocol was similar to NOPHO-AML 2004, and included the same two induction courses, but only three consolidation courses $\left(\mathrm{HA}_{2} \mathrm{E} 1, \mathrm{HA}_{3}\right.$, and $\mathrm{HA}_{2} \mathrm{E2}$, omitting $\left.\mathrm{HA}_{1} \mathrm{M}\right)$. Moreover, the protocol did not recommend HSCT in CR1 for any patient and did not include randomization to gemtuzumab ozogamicin. Children not in remission after two induction courses were taken off protocol. The protocols were approved by the national ethics committees in the eight participating countries and written informed consent was obtained from all patients or their parents or guardians.

\section{3 | Supportive care}

The NOPHO-AML 2004 protocol had recommendations on prophylactic sulfamethoxazole/trimethoprim 2-3 days per week. Prophylactic fluconazole was recommended until 1 month after the last chemotherapy course. No other prophylactic antibacterial or antiviral drugs were recommended. Use of prophylactic G-CSF was not generally recommended, but G-CSF could be considered in patients with neutropenia and life-threatening infection. The DB AML-01 protocol did not contain specific recommendations on prophylactic supportive care and thus prophylactic measures depended on local practice.

\subsection{Data collection}

Data on demographics, toxicity, and G-CSF administration (yes/no) after each course and follow-up information were registered prospectively in the joined database for the two protocols by the treating 
physician or local data manager. If data on G-CSF administration were missing for a course in the protocol database, it was assumed G-CSF was not given. If G-CSF administration was registered for a patient, an exploratory questionnaire was sent to the center where the patient was treated, retrospectively collecting information on G-CSF preparation, dose, treatment duration, and indication (infection, neutropenia without infection, prophylactic, or other). The indication was defined as prophylactic if G-CSF was administered within the first week after course start. If the daily dose of G-CSF was missing, the patient was assumed to have received the recommended dose for the cumulative dose analyses (filgrastim and lenograstim $5 \mu \mathrm{g} / \mathrm{kg} /$ day, pegfilgrastim $100 \mu \mathrm{g} / \mathrm{kg}$ given once). ${ }^{21,22}$

\section{5 | Definitions and statistics}

Standard deviation score of body mass index (BMI) for age and sex was calculated according to the World Health Organization growth standards. ${ }^{23}$ Chemotherapy dose reductions were defined as more than a $20 \%$ reduction of at least one drug dose or chemotherapy interruption of more than 7 days not including intrathecal treatment. Multiple logistic regression was used to assess the association of diagnostic year and G-CSF administration. Cox regression was used to assess the association between G-CSF administration and risk of relapse. For this analysis, follow-up started at the last day with absolute neutrophil count $<0.5 \times 10^{9} / \mathrm{L}$ after the last consolidation course $\left(\mathrm{HA}_{2} \mathrm{E} 2\right)$ and ended at relapse, death, or end of follow-up. This design excluded treatment-related mortality during therapy. Restricted cubic splines with three knots (at the 50th, 75th, and 90th percentiles) ${ }^{24}$ were used to show the continuous relationship of the cumulative dose of G-CSF and risk of relapse. Confounders incorporated into statistical models were defined as factors, which could be a cause of both independent and dependent variables, and were selected a priori. Country was included in the model of diagnostic year and G-CSF administration. Sex, age (continuous), BMI standard deviation score (continuous), $\mathrm{t}(8 ; 21)$ (yes/no), inv(16) (yes/no), KMT2A rearrangements (yes/no), FLT3/ITD status (FLT3/ITD yes/no), response to first induction ( $\geq 5 \%$ blasts between day 15 and start of second induction) (yes/no), and dose reductions (yes/no) were included as covariates in the adjusted model of G-CSF treatment and risk of relapse. Missing values for FLT3 status (24\%) were handled by multiple imputation for the analysis of G-CSF treatment and risk of relapse; for details, see Supplementary Material S1. All tests of significance were two sided. Statistical significance was defined as $P<0.05$.

\section{3 | RESULTS}

\section{1 | G-CSF treatment in NOPHO-AML 2004 and DB AML-01}

Of the 434 patients registered on NOPHO-AML 2004 and DB AML-01, five patients died before day 8 and 62 were treated with HSCT in CR1, and therefore excluded from this study (Figure 1). In total, 367 patients were included and 128 (35\%) received G-CSF as supportive care at least once during treatment (Figure 1). Four of the included patients received FLAG as a third induction course. Baseline characteristics for the cohort are shown in Table 1. Data on GCSF administration were missing in the protocol database after AIET in six $(2 \%)$ cases, after $\mathrm{AM}$ in four (1\%) cases, after $\mathrm{HA}_{1} \mathrm{M}$ in two (1\%) cases, after $\mathrm{HA}_{2} \mathrm{E} 1$ in 11 (3\%) cases, after $\mathrm{HA}_{3}$ in 10 (3\%) cases, and after $\mathrm{HA}_{2} \mathrm{E} 2$ in $12(4 \%)$ cases. In total, 1979 courses were given (not including GO and extra induction courses) and G-CSF was given after 269 (14\%) courses. In patients that received G-CSF at least once, 695 courses were given followed by G-CSF administration in 269 (39\%). Of the 367 patients in this study, 239 (65\%) never received G-CSF, 66 (18\%) received it after one course, 17 (5\%) after two, 27 (7\%) after three, eight (2\%) after four, four (1\%) after five, and six (2\%) received G-CSF after six courses of chemotherapy. The use of G-CSF differed between countries, but even more between centers-in centers that had treated 10 or more protocol patients, the percentage of patients that received G-CSF at least once ranged from 0 to $93 \%$. Of patients treated according to NOPHO-AML 2004, 39\% had received G-CSF at least once, and of patients treated according to DB AML-01, 23\% had received G-CSF at least once. G-CSF use was more common in patients treated between 2004 and 2009 and in patients with core binding factor leukemia (t[8;21] or inv[16]/t[16;16]), and less common in patients treated between 2010 and 2014, with FLT3/ITD and with KMT2A rearrangements (Table 1). The median cumulative neutrophil recovery time per course did not correlate with cytogenetic abnormalities or FLT3/ITD status (Supplementary Table S2). The odds ratio (OR) of G-CSF administration decreased with increasing diagnostic year (crude OR 0.9/diagnostic year, 95\% confidence interval [CI] 0.81.0; country-adjusted OR 0.8/diagnostic year, 95\% Cl 0.7-0.9).

In 19 patients with registered G-CSF use, all or some additional information was missing (Figure 1). The dose of G-CSF was missing after 11 courses. In the remaining cases, the median cumulative G-CSF dose was $75 \mu \mathrm{g} / \mathrm{kg}$ (range: 7-1460 $\mu \mathrm{g} / \mathrm{kg}$ ). The median total treatment duration was 13 days (range 1-85 days) in patients treated with filgrastim or lenograstim.

Table 2 shows information on G-CSF treatment per course. GCSF treatment was most common after $\mathrm{HA}_{1} \mathrm{M}$ and FLA-Dx and the most commonly used drug was filgrastim. The median daily dose of filgrastim or lenograstim was $5 \mu \mathrm{g} / \mathrm{kg}$ (range 3-12 $\mu \mathrm{g} / \mathrm{kg}$ ). The most common indication for treatment was infection. G-CSF treatment was usually started 2-3 weeks after the start of course if the indication was infection or neutropenia, but immediately after the end of chemotherapy if G-CSF was administered prophylactically (Table 2). The cumulative dose per course was higher if G-CSF was administered prophylactically.

\section{2 | G-CSF treatment and risk of relapse}

Of the included patients, 46 did not receive all chemotherapy courses due to leaving protocol $(n=4)$, relapse $(n=26)$, and death $(n=16$; not in CR1 [ $=8$ ]; in CR1 [ $=8]$ ). Of those that received and recovered from the last consolidation course $(n=321), 134$ (42\%) relapsed. All patients that received the last consolidation course were in complete remission after two induction courses and therefore none had 


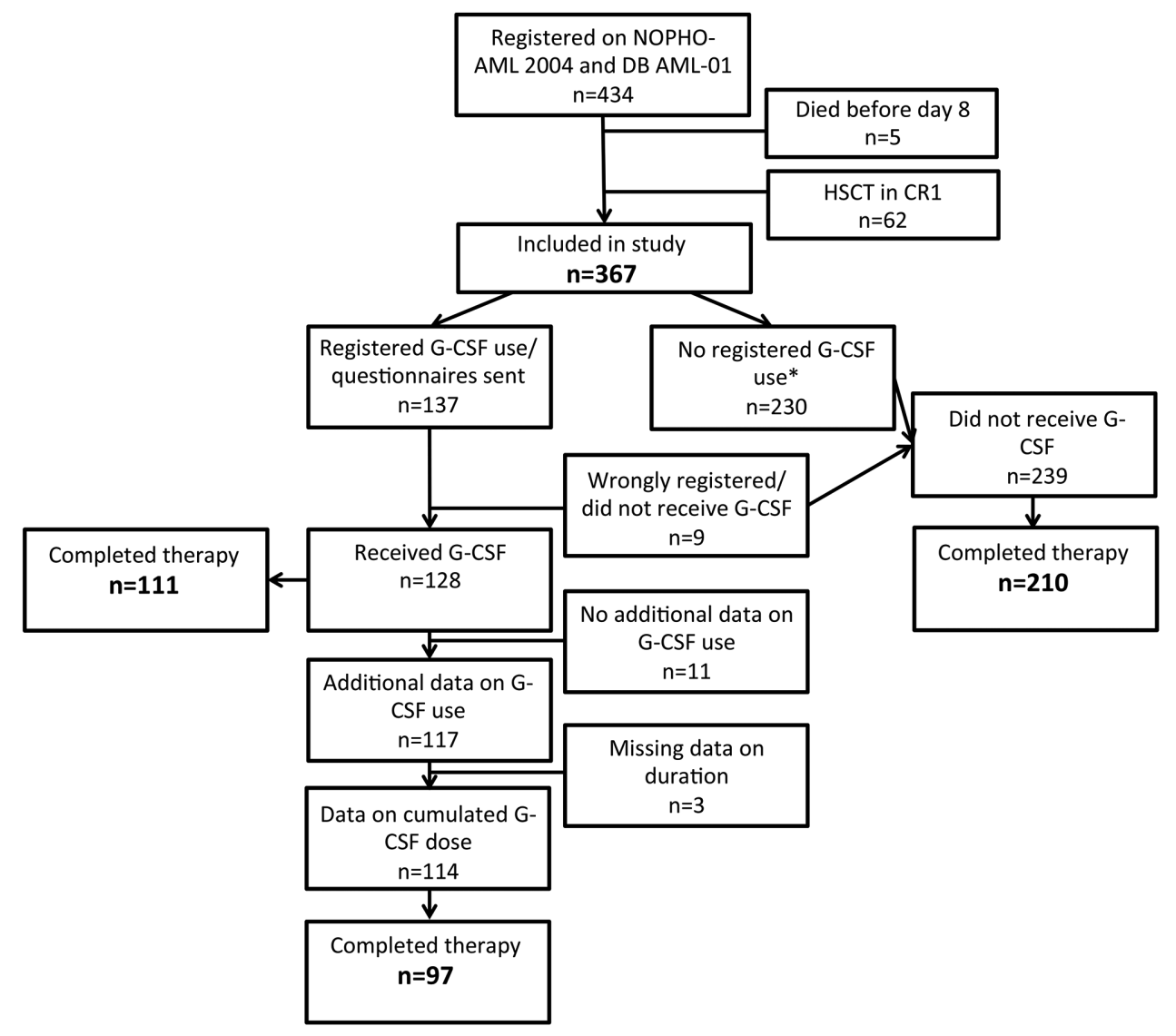

FIGURE 1 Flow diagram of included and G-CSF treated patients; G-CSF, granulocyte colony-stimulating factor; HSCT, hematopoietic stem cell transplantation; CR1, first complete remission. *Missing data on G-CSF administration in NOPHO database: after AIET in six (2\%) cases, after AM in four (1\%) cases, after $\mathrm{HA}_{1} \mathrm{M}$ in two (1\%) cases, after $\mathrm{HA}_{2} \mathrm{E} 1$ in $11(3 \%)$ cases, after $\mathrm{HA}_{3}$ in $10(3 \%)$ cases, and after $\mathrm{HA} \mathrm{E}_{2} \mathrm{E}$ in 12 (4\%) cases

received G-CSF (FLAG) as a part of treatment. The median follow-up time starting at recovery from last consolidation course for patients alive at last follow-up was 5.6 years (range 1.0-12.5 years). Patients that had received G-CSF at least once during treatment had higher risk of relapse compared to those who had not received G-CSF (5-year cumulative incidence of relapse: 51 vs $39 \%$, Table 3). Cumulative dose of G-CSF above the median did not increase the risk of relapse further (Table 3). In the continuous relationship of cumulative G-CSF dose and risk of relapse, it appears that a plateau in the risk of relapse was reached at a cumulative G-CSF dose of about 75-100 $\mu \mathrm{g} / \mathrm{kg}$ (Figure 2). Increasing doses above $100 \mu \mathrm{g} / \mathrm{kg}$ appeared to be associated with a slight decrease in risk of relapse, but the $\mathrm{Cl}$ is wide.

\section{4 | DISCUSSION}

In this large, population-based cohort including 367 unselected pediatric patients newly diagnosed with $\mathrm{AML}$ and treated in the Nordic countries, Hong Kong, the Netherlands, and Belgium from 2004 to 2013, we found that G-CSF was used as supportive care in about a third of patients, but the use decreased over time. There were large differences in G-CSF use across countries and centers. Filgrastim was the most commonly used drug and infection was the most common indication. Patients who had received G-CSF and finished treatment were at about $50 \%$ higher risk of relapse after adjustment for the most common and well-recognized risk factors.

G-CSF reduces the duration of neutropenia in children and adults treated for AML, but does not seem to reduce the risk of microbiologically documented infections or treatment-related mortality. ${ }^{13,14}$ Therefore, prophylactic G-CSF is not recommended in adults or children treated for AML. ${ }^{15,16}$ In the NOPHO-AML 2004 protocol, GCSF was not recommended for prophylactic use, but could be considered for patients with life-threatening bacterial or fungal infections. The DB AML-01 protocol did not include recommendations on G-CSF use. Despite the recommendations, a third of the patients received GCSF, though in the majority the indication was other than prophylactic. Restricting analysis to Nordic patients treated after 2009, about a fifth had received G-CSF (Table 1). This is comparable to a recent publication from the International Berlin-Frankfürt-Münster group showing that around a third of study groups use G-CSF in case of severe infection or prolonged neutropenia. ${ }^{25}$ Infection was the most common indication for G-CSF administration (Table 2), which is compatible with the fact that the frequency of severe infections was high for patients treated according to NOPHO-AML 2004. ${ }^{7}$ We have previously shown that $\mathrm{AIET}$ and $\mathrm{HA}_{1} \mathrm{M}$ were the most toxic courses and neutrophil recovery time was longer after AIET, FLA-Dx, and $\mathrm{HA}_{1} \mathrm{M},{ }^{7,26}$ which corresponds well with G-CSF being administered more frequently after these courses. More patients treated according to NOPHO-AML 
TAB LE 1 Baseline characteristics of the entire cohort and cohort stratified on G-CSF treatment

\begin{tabular}{|c|c|c|c|c|}
\hline & & \multirow[b]{2}{*}{$\begin{array}{l}\text { Entire cohort } \\
\mathrm{n}(\%)\end{array}$} & \multicolumn{2}{|c|}{ G-CSF treatment } \\
\hline & & & $\begin{array}{l}\text { Never } \\
\mathrm{n}(\%)\end{array}$ & $\begin{array}{l}\text { At least once } \\
\mathrm{n}(\%)\end{array}$ \\
\hline \multirow[t]{2}{*}{ Sex } & Male & $193(53)$ & $118(49)$ & $75(59)$ \\
\hline & Female & $174(47)$ & $121(51)$ & $53(41)$ \\
\hline BMI standard deviation score* & Median (range) & $0.0(-4.0-3.1)$ & $0.0(-4.0-3.1)$ & $0.0(-3.8-3.0)$ \\
\hline \multirow[t]{5}{*}{ Country } & Sweden & $91(25)$ & $61(26)$ & $30(23)$ \\
\hline & Denmark & $47(13)$ & $38(16)$ & $9(7)$ \\
\hline & Norway & $39(11)$ & $28(12)$ & $11(9)$ \\
\hline & Finland & $39(11)$ & $19(8)$ & $20(16)$ \\
\hline & Hong Kong & $45(12)$ & $11(5)$ & $34(27)$ \\
\hline \multirow[t]{2}{*}{ Protocol } & NOPHO-AML 2004 & $266(72)$ & $161(67)$ & $105(82)$ \\
\hline & DB AML-01 & $101(28)$ & $78(33)$ & $23(18)$ \\
\hline \multirow[t]{2}{*}{ Diagnostic year group } & 2004-2009 & $154(42)$ & $84(35)$ & $70(55)$ \\
\hline & $2010-2014$ & $213(58)$ & $155(65)$ & $58(45)$ \\
\hline \multirow[t]{2}{*}{ Diagnostic year group, Nordic countries only } & 2004-2009 & $137(62)$ & $81(54)$ & $56(79)$ \\
\hline & $2010-2013$ & $84(38)$ & $69(46)$ & $15(21)$ \\
\hline WBC $\left(10^{9} / L\right)$ at diagnosis* & Median (range) & $21(1-495)$ & $23(1-495)$ & $18(1-426)$ \\
\hline \multicolumn{2}{|c|}{$\geq 5 \%$ blast in bone marrow between day 15 and start of second induction* } & $87(24)$ & $56(23)$ & $31(24)$ \\
\hline \multicolumn{2}{|l|}{ FLA-Dx as second induction } & $39(11)$ & $28(12)$ & $11(9)$ \\
\hline $\begin{array}{l}\text { Treatment duration per course in days for those } \\
\text { who completed treatment }\end{array}$ & Median (range) & $30(19-47)$ & $29(19-46)$ & $31(22-47)$ \\
\hline \multicolumn{2}{|l|}{ Dose reductions of least one course ${ }^{\S}$} & $21(6)$ & $12(5)$ & $9(7)$ \\
\hline
\end{tabular}

BMI, body mass index; G-CSF, granulocyte colony-stimulating factor; ITD, internal tandem duplication; WBC, white blood count.

*BMI missing in three cases (0.8\%), WBC missing in one (0.3\%), cytogenetic abnormalities missing in two cases $(0.5 \%)$, bone marrow blasts after the first induction missing in six cases (2\%), and treatment duration missing in five cases.

${ }^{\S}$ More than $20 \%$ reduction of at least one drug dose or chemotherapy interruption of more than 7 days, intrathecal treatment not included.

2004 compared to patients treated according to DB AML-01 had received G-CSF (39 vs 23\%), which could be due to DB AML-01 being less toxic, but the two groups also differ in median diagnostic year and institutional practices.

Patients with core binding factor leukemia received G-CSF more often. Our group has previously shown that patients with $t(8,21)$ had inferior outcome before the second induction course was changed from AM to FLA-Dx in 2011 for patients with this cytogenetics abnormality. ${ }^{20}$ The model of G-CSF and risk of relapse included $t(8 ; 21)$ as a covariate. There was no difference in median cumulative neutrophil recovery time per course according to cytogenetic or FLT3/ITD status (Supplementary Table S2).
We found that G-CSF administration was associated with an increased risk of relapse in our cohort of patients. In adults with $A M L$, a large meta-analysis showed no association of G-CSF use and risk of relapse, ${ }^{13}$ but in children the association is not well studied. The pediatric AML protocol AML-BFM 98 included randomization to either no G-CSF or prophylactic G-CSF during induction and showed a trend for increased relapse risk in standard-risk patients treated with G-CSF. ${ }^{14}$ In a subsequent analysis of 50 standard risk patients treated according to the AML-BFM 98 protocol, it was found that patients overexpressing the differentiation-defective G-CSFR isoform IV treated with G-CSF had an increased risk of relapse. ${ }^{12}$ Our study cohort is comparable to the standard risk group of AML-BFM 98 in that we excluded patients 
TABLE 2 G-CSF treatment per course

\begin{tabular}{|c|c|c|c|c|c|c|c|c|c|}
\hline Coursoming & & AIET & AM & FLA-Dx & $\mathrm{HA}_{1} \mathrm{M}$ & $\mathrm{HA}_{2} \mathrm{E} 1$ & $\mathrm{HA}_{3}$ & $\mathrm{HA}_{2} \mathrm{E} 2$ & In total \\
\hline \multicolumn{2}{|c|}{ G-CSF administered, $\mathrm{n}(\%)^{\mathrm{a}}$} & $57(16)$ & $41(13)$ & $8(21)$ & $51(20)$ & $45(13)$ & $33(10)$ & $34(10)$ & $269(14)$ \\
\hline $\begin{array}{l}\text { Drug received, } \\
\text { n (\%) }\end{array}$ & Lenograstim & $1(2)$ & $2(5)$ & 0 & $3(6)$ & $1(2)$ & 0 & $3(9)$ & $10(4)$ \\
\hline \multicolumn{2}{|c|}{$\begin{array}{l}\text { Cumulative dose in } \mu \mathrm{g} / \mathrm{kg} \text {, } \\
\text { median (range) }\end{array}$} & $51(10-245)$ & $39(5-200)$ & $146(45283)$ & $43(5-540)$ & $50(5-220)$ & $53(5-200)$ & $55(5-200)$ & $75(7-1460)$ \\
\hline \multirow{2}{*}{$\begin{array}{l}\text { Indication for G-CSF, } \\
\mathrm{n}(\%)\end{array}$} & Infection & $35(61)$ & $18(44)$ & $6(75)$ & $22(43)$ & $15(33)$ & $10(30)$ & $12(35)$ & $118(44)$ \\
\hline & Neutropenia & $13(23)$ & $17(41)$ & $1(13)$ & $19(37)$ & $16(36)$ & $8(24)$ & $11(32)$ & $85(32)$ \\
\hline \multirow{3}{*}{$\begin{array}{l}\text { Course start to } \\
\text { G-CSF start, } \\
\text { median (range) }\end{array}$} & Infection & $17(6-32)$ & $15(0-31)$ & N/A & $18(13-37)$ & $15(10-26)$ & $15(3-19)$ & $15(7-16)$ & $16(0-37)$ \\
\hline & Neutropenia & $18(5-37)$ & $17(11-27)$ & $\mathrm{N} / \mathrm{A}$ & $18(6-35)$ & $15(4-31)$ & $14(3-40)$ & $13(6-35)$ & $16(2-40)$ \\
\hline & Prophylactic & N/A & N/A & N/A & N/A & $5(4-6)$ & $4(2-6)$ & N/A & $5(2-7)$ \\
\hline \multirow{3}{*}{$\begin{array}{l}\text { Cumulative dose in } \\
\mu \mathrm{g} / \mathrm{kg} \text { per course, } \\
\text { median (range) }\end{array}$} & Infection & $50(10-145)$ & $43(5-120)$ & $\mathrm{N} / \mathrm{A}$ & $35(5-540)$ & $40(15-175)$ & $40(15-125)$ & $30(5-157)$ & $43(5-540)$ \\
\hline & Neutropenia & $63(20-150)$ & $30(5-75)$ & N/A & $45(5-140)$ & $38(5-150)$ & $53(5-115)$ & $60(20-200)$ & $45(5-202)$ \\
\hline & Prophylactic & $N / A$ & $N / A$ & N/A & $\mathrm{N} / \mathrm{A}$ & $107(80-220)$ & $100(28-200)$ & N/A & $100(5-395)$ \\
\hline
\end{tabular}

G-CSF, granulocyte colony-stimulating factor; N/A, not applicable (median only stated for seven or more cases).

${ }^{a}$ Data on G-CSF administration were missing in the protocol database after AIET in six (2\%) cases, after AM in four (1\%) cases, after $\mathrm{HA} \mathrm{A}_{1} \mathrm{M}$ in two (1\%) cases, after $\mathrm{HA}_{2} \mathrm{E} 1$ in $11(3 \%)$ cases, after $\mathrm{HA}_{3}$ in $10(3 \%)$ cases, and after $\mathrm{HA}_{2} \mathrm{E} 2$ in $12(4 \%)$ cases. In case of missing data in the protocol database, it was assumed that the patient did not receive G-CSF.

${ }^{b}$ After seven courses, patients received pegfilgrastim followed by filgrastim (two patients after AIET, one patient after FLA-Dx, one patient after HA $\mathrm{A}_{1} \mathrm{M}$, two patients after $\mathrm{HA}_{2} \mathrm{E} 1$, and one patient after $\mathrm{HA}_{3}$ ).

'Other indications cover severe abdominal toxicity and G-CSF given to hasten marrow recovery.

TAB LE 3 G-CSF treatment and risk of relapse

\begin{tabular}{|c|c|c|c|c|c|c|}
\hline & $\begin{array}{l}\text { Patients who } \\
\text { finished } \\
\text { treatment (n) }\end{array}$ & $\begin{array}{l}\text { Five-year cumulative } \\
\text { incidence of relapse } \\
(95 \% \mathrm{Cl})\end{array}$ & $\begin{array}{l}\text { Crude HR } \\
(95 \% \mathrm{CI})\end{array}$ & $P$-value & $\begin{array}{l}\text { Adjusted }^{b} \text { HR } \\
(95 \% \mathrm{CI})\end{array}$ & $P$-value \\
\hline Never G-CSF treated & 210 & $39 \%(32-45 \%)$ & 1 & - & 1 & - \\
\hline $\mathrm{G}-\mathrm{CSF} \leq 75 \mu \mathrm{g} / \mathrm{kg}^{\mathrm{a}}$ & 49 & $52 \%(37-66 \%)$ & $1.5(0.9-2.3)$ & 0.09 & $1.5(1.0-2.5)$ & 0.07 \\
\hline G-CSF $>75 \mu \mathrm{g} / \mathrm{kg}^{\mathrm{a}}$ & 48 & $47 \%(32-62 \%)$ & $1.2(0.8-2.0)$ & 0.4 & $1.4(0.8-2.3)$ & 0.2 \\
\hline
\end{tabular}

$\mathrm{Cl}$, confidence interval; G-CSF, granulocyte colony-stimulating factor; HR, hazard ratio.

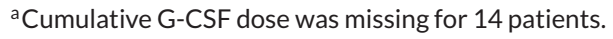

${ }^{b}$ Adjusted for sex, age, BMI standard deviation score, FLT3/ITD status, KMT2A rearrangements, inv(16), t(8;21), response to first induction ( $\geq 5 \%$ blasts between day 15 and start of the second induction course [yes/no]), and any dose reductions (yes/no).

treated with HSCT. The report of AML-BFM 98 does not address cumulative doses of G-CSF given, but patients received $5 \mu \mathrm{g} / \mathrm{kg} / \mathrm{day}$ from day 15 of first and second induction course and continued until the absolute neutrophil count exceeded $0.5 \times 10^{9} / \mathrm{L}$ on 3 consecutive days. Median time in neutropenia after the first induction course was 18 days and after the second induction course 11 days for G-CSF treated and therefore we estimate the median cumulative dose to be $(18+11)$ $\times 5=145 \mu \mathrm{g} / \mathrm{kg}$. This is twice as much as the median cumulative dose of our study, but comparable to that of those patients in our study that received G-CSF prophylactically. Figure 2 indicates that the increasing risk of relapse with increasing median cumulative G-CSF dose plateaus at $75-100 \mu \mathrm{g} / \mathrm{kg}$. Few patients were treated with doses over this level, hence statistical power to find the plateau threshold is limited. The fact that a plateau is reached corresponds with a study by Inaba et al showing that higher doses of filgrastim (10 vs $5 \mu \mathrm{g} / \mathrm{kg} /$ day) did not further decrease time in neutropenia. ${ }^{27}$ If higher doses of G-CSF do not 


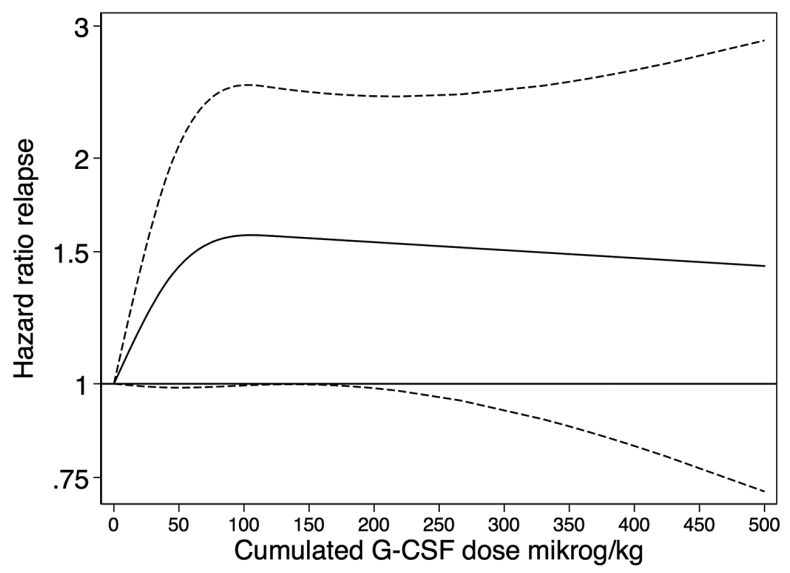

FIGURE 2 Cumulative G-CSF dose and risk of relapse; the figure shows the relationship of cumulative dose of G-CSF in $\mu \mathrm{g} / \mathrm{kg}$ and hazard ratio for relapse (solid line) with 95\% confidence intervals (dotted lines). Patients that received G-CSF but with missing cumulative dose $(n=14)$ were excluded from this analysis. Estimates are adjusted for sex, age (continuous), BMI standard deviation score (continuous), FLT3/ITD status (FLT3/ITD yes/no/missing), response to first induction ( $\geq 5 \%$ blasts from day 15 to start of second induction yes/no), and any dose reductions (yes/no). Only three patients received cumulative G-CSF dose above $500 \mu \mathrm{g} / \mathrm{kg}$, and therefore the $\mathrm{x}$-axis is terminated at $500 \mu \mathrm{g} / \mathrm{kg}$. G-CSF, granulocyte colony-stimulating factor

stimulate neutrophil proliferation further, it is possible that the same is true for AML blasts.

This study needs to be interpreted in light of its limitations. The frequency of G-CSF administration could be underestimated because in case of missing registration in the protocol database, it was assumed that the patient had not received G-CSF. However, since registration was close to complete ( $<5 \%$ missing), we believe this potential bias to be small. Also, with our nonrandomized study design, it was difficult to determine whether patients relapsed due to G-CSF treatment or were treated with G-CSF due to poor general condition, which could be associated with relapse. However, we incorporated the most common, well-established, and potential risk factors for relapse (sex, age, BMI standard deviation score, $\mathrm{t}(8 ; 21)$, inv(16), KMT2A rearrangements, FLT3/ITD status, response to first induction, and dose reductions) in the statistical model and the association remained. Nevertheless, we acknowledge the potential for residual confounding, since it would be impossible to incorporate into a model all the factors that physicians use in the clinical decision of whether G-CSF should be given. Follow-up began at the end of treatment, which separates exposure and outcome in time, avoiding the confounding factor that imminent relapse could result in slow bone marrow regeneration leading to G-CSF administration.

In conclusion, we found that supportive-care use of G-CSF was frequent for patients treated on NOPHO-AML 2004 and DB AML-01 with a large variation between centers. The use of G-CSF decreased over time. Our results indicate that children that received G-CSF as supportive care were at an increased risk of relapse and support the concern that G-CSF use might be harmful in pediatric AML.

\section{ACKNOWLEDGMENTS}

The authors would like to thank all the participating patients, parents, clinicians, and research staff at the NOPHO-DBH institutions. This study was supported by grants from the Danish Childhood Cancer Foundation, the Danish Cancer Society, and the Novo Nordisk Foundation.

\section{AUTHOR CONTRIBUTIONS}

D.J.A.L., P.H.A., and H.H. designed the project, interpreted the data, and drafted the manuscript; D.J.A.L. performed statistical analyses; J.A., G.J.L.K., S.-Y.H., O.G.J., M.K., B.L., B.D.M., J.P., and B.Z. collected the data and contributed to the writing of the manuscript.

\section{CONFLICTS OF INTEREST}

The authors have no conflicts of interest to report.

\section{ORCID}

Ditte J. A. Løhmann (D) https://orcid.org/0000-0002-9935-9157

\section{REFERENCES}

1. Gamis AS, Alonzo TA, Meshinchi S, et al. Gemtuzumab ozogamicin in children and adolescents with de novo acute myeloid leukemia improves event-free survival by reducing relapse risk: results from the randomized phase III Children's Oncology Group trial AAML0531. J Clin Oncol. 2014;32(27):3021-3032.

2. Pession A, Masetti R, Rizzari C, et al. Results of the AIEOP AML 2002/01 multicenter prospective trial for the treatment of children with acute myeloid leukemia. Blood. 2013;122(2):170-178.

3. Rubnitz JE, Inaba H, Dahl G, et al. Minimal residual disease-directed therapy for childhood acute myeloid leukaemia: results of the AML02 multicentre trial. Lancet Oncol. 2010;11(6):543-552.

4. Gibson BES, Webb DKH, Howman AJ, et al. Results of a randomized trial in children with acute myeloid leukaemia: Medical Research Council AML12 trial. Br J Haematol. 2011;155(3):366-376.

5. Tsukimoto I, Tawa A, Horibe K, et al. Risk-stratified therapy and the intensive use of cytarabine improves the outcome in childhood acute myeloid leukemia: the AML99 trial from the Japanese Childhood AML Cooperative Study Group. J Clin Oncol. 2009;27(24):4007-4013.

6. Creutzig U, Zimmermann M, Bourquin J-P, et al. Randomized trial comparing liposomal daunorubicin with idarubicin as induction for pediatric acute myeloid leukemia: results from study AML-BFM 2004. Blood. 2013;122(1):37-43.

7. Løhmann DJA, Abrahamsson J, Ha S-Y, et al. Effect of age and body weight on toxicity and survival in pediatric acute myeloid leukemia: results from NOPHO-AML 2004. Haematologica. 2016;101(11):13591367.

8. Sung L, Lange BJ, Gerbing RB, Alonzo TA, Feusner J. Microbiologically documented infections and infection-related mortality in children with acute myeloid leukemia. Blood. 2007;110(10):3532-3539.

9. Lehrnbecher T, Welte K. Haematopoietic growth factors in children with neutropenia. Br J Haematol. 2002;116(1):28-56.

10. Lowenberg B, Touw IP. Hematopoietic growth factors and their receptors in acute leukemia. Blood. 1993;81(2):281-292.

11. Souza LM, Boone TC, Gabrilove J, et al. Recombinant human granulocyte colony-stimulating factor: effects on normal and leukemic myeloid cells. Science. 1986;232(4746):61-65. 
12. Ehlers S, Herbst C, Zimmermann M, et al. Granulocyte colonystimulating factor (G-CSF) treatment of childhood acute myeloid leukemias that overexpress the differentiation-defective G-CSF receptor isoform IV is associated with a higher incidence of relapse. J Clin Oncol. 2010;28(15):2591-2597.

13. Gurion R, Belnik-Plitman Y, Gafter-Gvili A, et al. Colony-stimulating factors for prevention and treatment of infectious complications in patients with acute myelogenous leukemia. Cochrane Database Syst Rev. 2012;(6):CD008238.

14. Lehrnbecher T, Zimmermann M, Reinhardt D, Dworzak M, Stary J, Creutzig U. Prophylactic human granulocyte colony-stimulating factor after induction therapy in pediatric acute myeloid leukemia. Blood. 2007;109(3):936-943.

15. Döhner H, Estey E, Grimwade D, et al. Diagnosis and management of AML in adults: $2017 \mathrm{ELN}$ recommendations from an international expert panel. Blood. 2017;129(4):424-447.

16. Creutzig U, van den Heuvel-Eibrink MM, Gibson B, et al. Diagnosis and management of acute myeloid leukemia in children and adolescents: recommendations from an international expert panel. Blood. 2012;120(16):3187-3205.

17. Abrahamsson J, Forestier E, Heldrup J, et al. Response-guided induction therapy in pediatric acute myeloid leukemia with excellent remission rate. J Clin Oncol. 2011;29(3):310-315.

18. Hasle H, Abrahamsson J, Forestier E, et al. Gemtuzumab ozogamicin as postconsolidation therapy does not prevent relapse in children with AML: results from NOPHO-AML 2004. Blood. 2012;120(5):978-984.

19. De Moerloose B, De Bont ESJM. The Dutch-Belgian pediatric acute myeloid leukemia protocol for children with newly diagnosed acute myeloid leukemia (DB AML-01). Belg J Hematol. 2010;1(1):32-34.

20. Hasle H, Abrahamsson J, De Bont ES, et al. Anthracycline type during induction associated with outcome in pediatric $\mathrm{t}(8 ; 21)$ and inv(16) AML. Blood. 2014;124(21):11. [abstract].

21. Schaison G, Eden OB, Henze G, et al. Recommendations on the use of colony-stimulating factors in children: conclusions of a European panel. Eur J Pediatr. 1998;157(12):955-966.
22. André N, Kababri ME, Bertrand $P$, et al. Safety and efficacy of pegfilgrastim in children with cancer receiving myelosuppressive chemotherapy. Anticancer Drugs. 2007;18(3):277-281.

23. de Onis M, Lobstein T. Defining obesity risk status in the general childhood population: which cut-offs should we use? Int J Pediatr Obes. 2010;5(6):458-460.

24. Durrleman S, Simon R. Flexible regression models with cubic splines. Stat Med. 1989;8(5):551-561.

25. Klein K, Hasle H, Abrahamsson J, De Moerloose B, Kaspers GJL. Differences in infection prophylaxis measures between paediatric acute myeloid leukaemia study groups within the International BerlinFrankfürt-Münster (I-BFM) study group. Br J Haematol. 2018;183:8795.

26. Løhmann DJA, Asdahl PH, Abrahamsson J, et al. Associations between neutrophil recovery time, infections and relapse in pediatric acute myeloid leukemia. Pediatr Blood Cancer. 2018;65(9):e27231.

27. Inaba H, Cao X, Pounds $\mathrm{S}$, et al. Randomized trial of 2 dosages of prophylactic granulocyte-colony-stimulating factor after induction chemotherapy in pediatric acute myeloid leukemia. Cancer. 2010;117(6):1313-1320.

\section{SUPPORTING INFORMATION}

Additional supporting information may be found online in the Supporting Information section at the end of the article.

How to cite this article: Løhmann DJA, Asdahl PH, Abrahamsson $\mathrm{J}$, et al. Use of granulocyte colony-stimulating factor and risk of relapse in pediatric patients treated for acute myeloid leukemia according to NOPHO-AML 2004 and DB AML-01. Pediatr Blood Cancer. 2019;e27701. https://doi.org/ 10.1002/pbc.27701 